\title{
Farmacoeconomia e malattie cardiovascolari
}

\author{
Lorenzo G. Mantovani $^{(1)}$, Andrea Morsanutto ${ }^{(2)}$, Simona de Portu ${ }^{(1)}$
}

Alla base della nascita di una disciplina come la farmacoeconomia si trovano quelli che nel mondo anglosassone sono chiamati gli unavoidable facts of life, ossia i fatti inevitabili della vita che non riguardano soltanto i sistemi sanitari, e il SSN italiano in particolare, ma, più in generale, l'organizzazione delle nostre società.

Le nostre società affrontano ogni giorno la richiesta di più interventi sanitari di quanti ne possano finanziare, e ciò anche eliminando gli sprechi: potenzialmente, a livello del singolo individuo, gli interventi sanitari sono infiniti, ma le risorse sono limitate.

La conseguenza di questa aporia è che le risorse dedicate a un certo intervento sanitario sono necessariamente sottratte ad altro.

Ciò pone quindi la necessità che qualcuno prenda decisioni su che cosa vada finanziato, decisioni che devono essere supportate da strumenti e criteri sulla base dei quali scegliere.

Il problema farmacoeconomico potrebbe sembrare nuovo, ma se, sicuramente, è recente l'evidenza della sua estensione, si tratta in realtà di una condizione da sempre connaturata alle scelte in tema sanitario.

In passato, e anche in Italia fino alla creazione del SSN con le casse mutue e le successive riforme, il problema farmacoeconomico era confinato all'ambito individuale.

Non era un problema sociale e politico ma di censo individuale: chi poteva pagare aveva accesso alle cure, chi non poteva pagare ne restava escluso.

Con l'assunzione del costo sanitario da parte delle nostre società, emerge la necessità di definire un criterio, di fondare oggettivamente la scelta sul cosa, chi, quando e come trattare.

Non è certo etico lasciare al "caso" questa scelta, come accadrebbe lasciando che sia la disponibilità di budget nel momento dell'erogazione a decidere del trattamento.

Di fronte al rischio che sia "il caso" a governare il futuro dei nostri Pazienti, dobbiamo cercare criteri che ci possano aiutare, che siano razionali nel contesto del sistema sanitario di un Paese moderno.

Nel 1977 [1] due autori, Weinstein e Stason, hanno affrontato per la prima volta in maniera sistematica questo problema, sulle pagine del New England Journal of Medicine, in un articolo dal titolo Foundations of cost-effectiveness analysis for health and medical practices.

Non è un caso che si sia dovuto attendere fino ad allora: gli anni ' 70 rappresentano, per motivi economici e demografici, un punto di cesura rispetto all'esperienza precedente di gestione della spesa pubblica in generale e sanitaria in particolare.

Le ragioni della crescita della spesa sanitaria sono legate a una fortunata tendenza demografica: la popolazione invecchia sempre di più; se agli inizi del ventesimo secolo l'aspettativa di vita era inferiore a 50 anni per uomini e donne, nel 2000 questa è divenuta di circa 81 anni per le donne e 75/76 anni per gli uomini, quasi raddoppiando.

Questa circostanza ha un corollario importante dal punto di vista farmacoeconomico: le malattie tendono ad essere più frequenti nelle persone anziane e gli anziani tendono a utilizzare i servizi sanitari, cioè a creare delle spese, in maggior quantità.

A fianco dell'allungamento dell'età media si pone l'evoluzione tecnologica: vengono introdotte nuove tecnologie che sono più efficaci e che aumentano la nostra attesa di vita ma, almeno nel breve periodo, sono normalmente anche più costose.

Un esempio può essere quello della terapia trombolitica: la mortalità intraospedaliera per infarto senza trombolisi si aggira intorno al $15 \%$, post-trombolisi scende sotto il $10 \%$.

Questo vuol dire che ogni 100 soggetti che arrivano in ospedale vivi, ne sono dimessi 5 in più, cioè 5 persone in più che potranno invecchiare, che avranno necessità di assistenza sanitaria e che sono a rischio di ictus o di sviluppare scompenso, un'attesa e un rischio che, a loro volta, generano la necessità dell'utilizzo di nuove tecnologie sanitarie.

L'insieme di questi fattori interagisce, dando luogo a quella che potremmo definire una "storia di successo".

È un successo sicuramente importante per le nostre società, per la nostra aspettativa di vita $\mathrm{e}$ per la qualità della nostra vita futura, ma anche una storia costosa.

\author{
(1) CIRFF, Centro \\ Interdipartimentale \\ di Ricerca in \\ Farmacoeconomia e \\ Farmacoutilizzazione, \\ Università degli Studi \\ di Napoli Federico II \\ (2) Servizio Assistenza \\ Farmaceutica, \\ Direzione Centrale \\ Salute e Protezione \\ Sociale, Regione Friuli \\ Venezia Giulia
}


Le valutazioni di natura economica applicate ai farmaci e, in generale, alle terapie preventive, diagnostiche, alla cura e alla riabilitazione dei malati e delle malattie, le facciamo perché ci aiutino a prendere la decisione su "chi riceverà cosa", ma sono solo un elemento della decisione.

La parte più importante, infatti, è il valore perseguito, nel nostro caso indicato dal disposto dell'articolo 32 della Costituzione, per il quale "la Repubblica tutela la salute come fondamentale diritto dell'individuo e interesse della collettività".

Questo significa qualità, sicurezza ed efficacia di prevenzione e trattamento delle malattie, l'elemento centrale a cui aggiungiamo, nel momento in cui ci occupiamo di programmi pubblici, anche i costi.

Non si tratta di "razionare" le risorse, bensì di razionalizzarne l'utilizzo, di cercare di massimizzare il benessere, la salute che deriva dall'usare risorse che sono scarse, che possiamo destinare a interventi o a pazienti differenti.

Confrontare costi ed effetti o costi e benefici significa, nel nostro caso, confrontarci con il miglioramento della salute indotto dagli interventi adottati, e per fare questo misuriamo degli eventi che, se la salute migliora, normalmente evitiamo.

Ad esempio evitiamo degli eventi cardiovascolari, evitiamo che il soggetto perda l'attività lavorativa.

Ovviamente avremo dei costi, legati in generale al trattamento e alla gestione degli eventi avversi, e avremo anche dei risparmi.

Queste premesse non risolvono però la domanda su come valutare costi e benefici in ambito sanitario.

Sovvengono essenzialmente tre tipi di tecniche: l'analisi di costo/efficacia, l'analisi di costo/utilità e l'analisi di costo/beneficio.

L'Analisi Costo/Efficacia è la più utilizzata e confronta il costo di un intervento con la misurazione di parametri biochimici o clinici oppure di eventi (i cosiddetti hard end-points) quali ad esempio la riduzione della mortalità e il prolungamento della sopravvivenza.

Lo strumento, cioè, indica quanto costa prolungare la sopravvivenza di un certo individuo di un anno, offrendo il "costo per anno di vita guadagnato".

Un'evoluzione di questa tecnica si pone il problema non solo della quantità di vita ottenuta, ma della qualità di vita risultante.

È la tecnica dell'Analisi Costo/Utilità, che nasce in oncologia di fronte al trade off specifico del miglioramento della performance di sopravvivenza di un soggetto: possiamo migliorare la quantità di vita di un soggetto con la chemioterapia, ma possiamo anche peggiorarne la qualità in maniera notevole, e allora bisogna scegliere se prolungare la sopravvivenza, magari di “po- che" settimane al prezzo di un peggioramento significativo della qualità, oppure no.

$\mathrm{Su}$ tale postulato si è cominciato ad elaborare un indice sintetico, che cioè pesa la lunghezza della vita in funzione di quanto sia desiderabile vivere in certe condizioni per quella che gli economisti chiamano "utilità"; ciò che ne consegue è l'indice sintetico che si chiama QALY (Quality Adjusted Life Year).

Per vedere come il concetto di QALY trovi concretezza, possiamo fare riferimento all'esperienza britannica, in cui il NICE (National Institute of Clinical Excellence) prende decisioni sulla raccomandazione circa l'utilizzo di tecnologie sanitarie utilizzando il QALY come parametro: analizzando le decisioni di questa organizzazione, vediamo che gli interventi sono solitamente considerati costo/efficaci quando il loro costo è inferiore alle 30-35.000 sterline per QALY, e ne viene quindi raccomandato l'utilizzo, mentre gli interventi con un costo superiore a questo importo non sono considerati costo/ efficaci, e quindi non ne viene raccomandato l'utilizzo. Questo limite delle 30-35.000 sterline nasce, in maniera pragmatica, dall'esperienza in materia di dialisi.

Infine abbiamo l'Analisi Costo/Beneficio, che è stato il primo tipo di valutazione condotto ma che è anche il meno utilizzato perché mette a confronto il denaro con la sopravvivenza, sia pure sopravvivenza aggiustata per la qualità, il che significa dare un esplicito peso monetario alla vita umana o ai suoi aspetti.

Si tratta di un procedimento tecnicamente complesso, anche se esistono tecniche elaborate di recente, importate da aree dell'economia differenti come i trasporti e l'ambiente, che lo rendono più semplice.

È eticamente e, più ancora, politicamente complesso.

L'essenza di questo metodo è il confronto tra le vite che siamo in grado di salvare con un certo tipo di intervento e quelle che siamo in grado di salvare con un altro intervento: dal punto di vista economico è la stessa cosa, il denaro non è un valore in sé, ma è considerato - in sanità - in funzione delle vite che possiamo salvare.

Questo tipo di scelte, cioè fra vite degli uni e vite degli altri, viene costantemente operato all'interno dei nostri sistemi perché ogni volta, per esempio, che un'autorità sanitaria decide, avendo delle risorse da dedicare ad uno screening oncologico, di dedicarlo a un tumore piuttosto che a un altro, sta scegliendo e sta dando un valore relativo alle vite degli uni rispetto alle vite degli altri.

Ciò che rende comunemente accettato questo confronto è il fatto che non è diretto, è implicito; è il renderlo esplicito a divenire, nel sentire comune, "scandaloso". 


\section{APPROCCIO FARMACOECONOMICO PER LA PRIMA CAUSA DI MORTE NEL MONDO}

In Italia, circa il $42-43 \%$ delle morti è attribuibile alle malattie cardiovascolari, seguono i tumori, con una percentuale di circa $1 / 4$, e poi tutto il resto.

Questo dato spiega quanto le malattie cardiovascolari siano rilevanti dal punto di vista sanitario, resta ora da chiarirne il significato economico.

\section{ALCUNI STUDI SUI COSTI DELLA MALATTIA CARDIOVASCOLARE IN ITALIA}

Nel 2005 è stata presentata al congresso dell'International Society for Pharmacoeconomics and Outcomes Research l'analisi interinale condotta su uno studio [2] sulla stima della prevalenza dei fattori di rischio cardiovascolari in soggetti di età fra i 40 e i 79 anni.

Settecento medici di medicina generale, appartenenti alla Società Italiana di Medicina Generale, si sono impegnati ad arruolare, in base ad un algoritmo casualizzante, 16 soggetti ciascuno; per ciascuno di questi soggetti è compilata una scheda di raccolta dati informatizzata, e sono effettuati un prelievo di sangue e altre procedure.

Al completamento dello studio saranno stati arruolati 11.000 soggetti.

L'analisi interinale è stata condotta quando lo studio aveva arruolato poco meno di 4.000 soggetti.

Su questi 4.000 sono stati individuati 197 coronaropatici, appaiati per età e sesso a 197 controlli.

L'età media era di 65,3 anni $( \pm 8,8)$ e il gruppo era composto per circa il $70 \%$ da maschi, con questa ripartizione delle malattie coronariche: il $35,5 \%$ aveva l'angina, il $23,9 \%$ un pregresso infarto, il 2,5\% una rivascolarizzazione, il 38,1\% due o più degli eventi suddetti, e ciò con una prevalenza omogenea con quella riscontrabile nel "progetto cuore" dell'ISS (40-49 aa: 1\%; 50-59 aa: $4,2 \%$; 60-69 aa: $6,7 \% ; 70-79$ aa: $11,6 \%)$

Da questo studio osserviamo che, in media, il paziente coronaropatico costa al SSN 351,7 euro/mese, rispetto ai 110 euro/mese per il controllo e con una differenza altamente significativa di oltre 241 euro/mese.

Nell'elaborazione dei dati è stata utilizzata la tecnica statistica nota come bootstrap, un metodo di regressione con simulazione che serve per calcolare gli intervalli di confidenza in presenza di distribuzioni sulle quali non sarebbe possibile fare le classiche ipotesi di una regressione lineare.

Un'altra osservazione che è possibile fare riguarda il gradiente in funzione dell'età: il costo nei coronaropatici, infatti, tende a decre- scere con il crescere dell'età, nel momento in cui altre malattie si fanno più frequenti nei non coronaropatici, per cui la differenza è massima nei soggetti più giovani, sotto i 60 anni, ed è minima nei soggetti settantenni.

Questo fenomeno è probabilmente legato a un effetto di sopravvivenza, il cosiddetto healthy survivor effect, per il quale a 75-79 anni arrivano soltanto i soggetti che erano i "migliori" coronaropatici, mentre gli altri soggetti vengono persi prima.

Un altro studio ha assunto un approccio completamente diverso, in collaborazione con l'autorità sanitaria del Friuli Venezia Giulia.

Si tratta di uno studio retrospettivo, longitudinale, basato sull'incidenza di costo delle malattie e che utilizza la base dati amministrativa della regione Friuli Venezia Giulia.

Sono stati considerati tutti i residenti della Regione dell'anno 2000, e sono stati studiati i soggetti che durante questo periodo hanno sviluppato una malattia cardiovascolare che porta a ricovero e che sono stati ospedalizzati per insufficienza cardiaca [3], ictus [4], infarto miocardico acuto [5]. È stato adottato il criterio di escludere i soggetti che avevano ospedalizzazione nei cinque anni precedenti, cioè tra il 1995 e il 1999.

I soggetti sono stati seguiti dal momento dell'evento, cioè dall'entrata in ospedale, fino al 31 dicembre 2004 o alla morte o alla revoca del consenso, qualunque evento accadesse per primo.

Da questi soggetti sono stati estratti [4] i 936 soggetti che avevano un'ospedalizzazione per ictus.

Si trattava più frequentemente individui di sesso femminile, con un'età avanzata intorno ai 77 anni; il costo medio dell'ictus per anno/ persona è di 3.500 euro circa, $3 / 4$ dei quali attribuibili alle ospedalizzazioni; questo non è il costo sociale, ma semplicemente il costo per la componente sanitaria. Il costo sociale per l'ictus è abbastanza elevato perché richiede spesso una gestione da parte dei familiari, ma in questa analisi era considerata solo la prospettiva del servizio sanitario regionale. Per quanto riguarda i costi, osserviamo che il primo anno si aggirano intorno a 9.000 euro, il che comprende $\mathrm{i}$ costi di ospedalizzazione, mentre il secondo, il terzo, il quarto e il quinto anno sono approssimativamente 2.000 euro.

I costi dei farmaci invece sono più elevati col passare del tempo, e ciò può essere spiegato considerando che aveva più probabilità di arrivare al quinto anno chi riceveva e tollerava (tutte) le terapie appropriate. Circa 1/3 dei pazienti è morto durante il follow-up, la sopravvivenza tende a decrescere nel tempo, in misura maggiore in funzione del gruppo di età di appartenenza.

Proporzioni analoghe sono derivabili nella sottopopolazione dei soggetti con infarto acuto 
del miocardio [5]: 1.185 soggetti ospedalizzati vivi, con un'età media di 71 anni e per il $60 \%$ donne; in questo caso il costo è stato di quasi 5.000 euro per anno/persona, così suddiviso: il $70 \%$ ospedalizzazioni, il $20 \%$ farmaci e la differenza, circa il 10\%, è tutto il resto. Quasi il $40 \%$ di coloro che sono arrivati vivi all'ospedale è morto durante il follow-up, con una frequenza maggiore, ovviamente, tra gli anziani.

I costi in termini di farmaci sono, di nuovo, all'inizio inferiori, poi tendono a crescere col passare del tempo; le ospedalizzazioni, analogamente, sono molto elevate il primo anno, perché c'è un evento e molto spesso c'è un secondo evento di ospedalizzazione, e poi decrescono con il passare del tempo.

Circa la sopravvivenza, i soggetti giovani arrivati in ospedale facevano registrare una sopravvivenza superiore all' $85 \%$ in media a quattro anni e mezzo, e anche in questa analisi la sopravvivenza calava con l'aumentare dell'età.

Un'esperienza condotta a Portogruaro [6] ha avuto come oggetto i pazienti diabetici, attraverso la revisione delle cartelle cliniche di circa 300 soggetti con un follow-up di circa 16 mesi e la valutazione dei costi sanitari diretti della patologia e di quelli dovuti alla presenza e alla prevalenza di complicanze.

In breve, il soggetto diabetico senza complicanze costa mediamente 1.039 euro, con una complicanza costa circa 2.000 euro, con due o più complicanze 3.141 euro.

\section{È COSTO/EFFICACE TRATTARE LE MALATTIE CARDIOVASCOLARI?}

La domanda sulla costo/efficacia del trattamento delle malattie cardiovascolari andrebbe posta in una maniera più specifica: "È costo/efficace trattare le malattie cardiovascolari in modo appropriato?". Un'analisi [7] sui dati cumulati di diversi studi condotti nell'arco degli ultimi 10 anni, dallo studio $4 \mathrm{~S}$ in poi, mostra che, su 1.000 soggetti trattabili in prevenzione primaria, trattati in maniera appropriata e continuativa con una statina, in funzione dell'efficacia della statina (cioè di quanto è in grado sostanzialmente di ridurre il colesterolo totale e LDL e di aumentare l'HDL), è possibile evitare tra $\mathrm{i} 70 \mathrm{e}$ i 110 eventi in 10 anni, dove il numero maggiore di eventi evitati corrisponde alle statine più efficaci, e quello minore alle statine meno efficaci.

Questo significa, per questa ipotetica coorte di 1.000 soggetti trattati per 10 anni, prevenire tra le 30 e le 50 morti circa o guadagnare tra $i$ 150 e i 250 anni di vita.

È possibile, su queste basi, fare una valutazione farmacoeconomica.

Operando una stima molto conservativa, possiamo calcolare una spesa intorno ai 4,5 milioni di euro per 10 anni per 1.000 pazienti, con un risparmio di circa mezzo milione di euro dovuto al calcolo dei costi in acuto degli eventi evitati, per una spesa quindi di circa 4 milioni di euro in 10 anni.

Ora, considerando che il trattamento fa guadagnare circa 200 anni di vita, possiamo indicativamente determinare il costo di prolungamento della sopravvivenza di un anno in circa 15-20.000 euro.

Questo anno, ottenuto in prevenzione primaria, è un LYG (anno di vita guadagnato), ma è anche un QALY perché il soggetto è sostanzialmente libero da malattia ed è sostanzialmente in perfetta salute.

Confrontando questo costo con il cut-off di 50.000 euro per QALY, è possibile affermare che il rapporto di costo/efficacia medio della terapia con statine è abbondantemente entro $\mathrm{i}$ limiti di ciò che è considerato in letteratura come costo/efficace in sanità.

È possibile anche trarre un'osservazione ulteriore, quella per cui tanto più è efficace la statina, tanto più è favorevole questo rapporto, tanto meno costa, tanto più è favorevole.

\section{QUANTI SOGGETTI POSSIAMO TRATTARE?}

Un'analisi condotta nel 2004 sui dati OsMed 2003 [8] valuta la spesa sostenuta dal SSN italiano per l'acquisto di statine.

All'epoca questa spesa era di 775 milioni di euro, che corrispondevano circa a 37 DDD (dose media giornaliera di farmaco impiegata nel trattamento di un adulto, di circa $70 \mathrm{~kg}$, con riferimento all'indicazione terapeutica principale) per 1.000 abitanti.

Considerando alcuni limiti specifici (la DDD delle statine è molto bassa e la persistenza al trattamento non è ottimale), questo investimento corrisponde a circa 500-550 milioni di giornate di terapia, ossia alla possibilità di trattare 1,5 milioni di individui in modo continuativo e appropriato per un anno intero. Ma se facciamo riferimento alla persistenza al trattamento, cioè quanto un soggetto che ha iniziato una terapia con statine la continua nel tempo, vediamo che, a 6 mesi, meno del $50 \%$ è ancora in terapia; questo almeno è il dato che emerge dalle analisi condotte a cavallo dello scorso decennio. Questo fenomeno di abbandono delle terapie croniche non è per altro peculiare delle statine. In una recente analisi sui farmaci anti-ipertensivi [9], si osservano delle differenze nella probabilità dei soggetti di continuare la terapia in funzione della classe di farmaci prescritta e di altre caratteristiche; ad esempio è notevole che i pazienti dei medici in località con meno di 50.000 abitanti mediamente persistono più a lungo rispetto a quelli che abitano in luoghi con più di 50.000 abitanti: probabilmente la relazione medico/paziente e forse anche il tempo da dedicare al paziente si confermano essere un elemento importante. 
Il problema di un'aderenza alla terapia estremamente bassa può essere legato a diversi fattori in grado di influenzarla: in parte, forse, allo stesso medico, in parte alla modalità di prescrizione del farmaco, al paziente, ma anche al sistema di controllo della spesa; verosimilmente esiste una serie di fattori che non è stata investigata estesamente.

Importante in questo senso è che la pratica quotidiana è diversa dai trial clinici, dove il paziente e il medico concludono un vero e proprio contratto, in cui il medico si impegna a curare il paziente nel modo migliore e questo sottoscrive il proprio consenso informato impegnandosi a sua volta. Inoltre al paziente coinvolto in un trial è dedicato parecchio tempo, fattore considerato dalla letteratura come il miglior predittore $\mathrm{di}$ persistenza alla terapia: nella pratica clinica reale il tempo è minore, e probabilmente il paziente recepisce in misura inferiore i motivi della prescrizione e la necessità della persistenza.

Se la persistenza è un tema fondamentale, altrettanto lo è quello dell'appropriatezza della prescrizione: lo studio CHECK mostra come tra i 197 soggetti arruolati, solo il 47,7\% era in terapia con un farmaco ipolipidemizzante mentre, in funzione della nota 13 e dell'evidenza dei trial clinici, e a meno di controindicazioni individuali, teoricamente il $100 \%$ dei soggetti avrebbe dovuto già essere trattato con statine.

Tornando alla domanda iniziale ("Possiamo permetterci di trattare i pazienti?"), i dati che abbiamo esaminato indicano una risposta affermativa, ma con modalità appropriate e forse, con le nuove funzioni di rischio, anche al di sotto del $2 \%$ annuo di rischio assoluto, soprattutto in alcune categorie d'individui, come i soggetti con meno di 60 anni, quelli che dal punto di vista economico rendono più evidente il differenziale tra prevenzione e non prevenzione, fra soggetto con eventi o soggetto libero da eventi.

In questi soggetti è razionale trattare, dal punto di vista clinico e forse anche dal punto di vista economico, perché dal punto di vista economico è possibile ottenere un risparmio maggiore.

\section{BIBLIOGRAFIA}

1. Weinstein MC, Stason WB. Foundations of cost-effectiveness analysis for health and medical practices. $N$ Engl $J$ Med 1977; 296: 716-21

2. de Portu S, Ronzini M, Mazzarotto E, Galietti M, Mantovani LG. Cost of cardiovascular disease: a case control study. Value Health 2005; 8: A2-A3

3. Morsanutto A, Mantovani LG, Ros B, de Portu S, Spazzapan D, Tosolini F. Costs and outcomes after first heart failure hospital admission: a longitudinal study using administrative databases. Value Health 2005; 8: A96-A97

4. Morsanutto A, Mantovani LG, Ros B, de Portu S, Spazzapan D, Tosolini F. Cost and outcomes after first stoke hospital admission: a longitudinal study using administrative databases. Value Health 2005; 8: A151-A152

5. Morsanutto A, Mantovani LG, Ros B, de Portu S, Spazzapan D, Tosolini F. Cost and outcomes after first acute myocardial infarction hospital admission: a longitudinal study using administrative databases. Value Health 2005; 8: A112

6. Morsanutto A, Berto P, Lopatriello S, Gelisio R, Voinovich D, Cippo PP, Mantovani LG. Major complications have an impact on total annual medical cost of diabetes: results of a database analysis. J Diabetes Complications 2006; 20: $163-9$

7. Dati del Centro di Farmacoeconomia dell'Università degli Studi di Milano

8. http://www.ministerosalute.it/medicinali/resources/documenti/osmed/rapporti/osmed_2003.pdf

9. Mazzaglia G, Mantovani LG, Sturkenboom MC, Filippi A, Trifirò G, Cricelli C, Brignoli O, Caputi AP. Patterns of persistence with antihypertensive medications in newly diagnosed hypertensive patients in Italy: a retrospective cohort study in primary care. J Hypertens 2005; 23: 2093-2100 